\title{
Identificação e descrição da brincadeira em uma espécie pouco estudada, o boto cinza (Sotalia fluviatilis), em seu ambiente natural
}

\author{
Luísa Helena Pinheiro Spinelli \\ Lídio França do Nascimento \\ Maria Emília Yamamoto \\ Universidade Federal do Rio Grande do Norte
}

\begin{abstract}
Resumo
Comportamentos de brincadeira são fáceis de identificar, mas difíceis de definir. Sua ocorrência é mais freqüente em animais jovens, apresentando algumas características comuns: falta de função aparente, utilização de comportamentos específicos da espécie de forma exagerada em intensidade ou número de repetições. Estudamos a brincadeira em uma espécie pouco conhecida, o boto cinza, durante 18 meses, na Praia de Pipa, RN. Definimos seis categorias de brincadeira: com objeto, de forrageio, locomotora, surf, social e de impulsão. Os comportamentos descritos ocorreram quando havia filhotes nos grupos observados. Sugerimos que alguns tipos de brincadeira possibilitam o exercício da musculatura, enquanto que outras também permitem o desenvolvimento de relações sociais. A brincadeira com objetos e de forrageio treinam o animal para a pesca, habilidade complexa que deve dominar antes de se tornar independente. Nossos dados sugerem que, à semelhança dos mamíferos terrestres, nos mamíferos aquáticos a brincadeira tem função adaptativa, preparando o indivíduo para a vida adulta.
\end{abstract}

Palavras-chave: Boto cinza, Brincadeira, Habilidades de forrageio, Animais imaturos.

\begin{abstract}
Identification and description of play in a scarcely studied species, the tucuxi (Sotalia fluviatilis) in a field site

Play behavior are easy to identify but difficult to define. It is more frequently displayed by young animals and present some distinguished characteristics: no apparent function and exaggerated repetition or intensity of species-specific behaviors. We studied play behavior in a scarcely studied species, the tucuxi, during 18 months at Pipa, RN, Brazil . We defined six categories: object, foraging, locomotor, social and impulsion play plus surf. The behaviors described occurred only when immatures were present in the observed groups. We suggest that some kinds of play exercise the muscles while others also allow for the development of social interactions. Object and foraging play are opportunities for training of fishing abilities which the animal must master before becoming independent. Our data suggest that, like terrestrial mammals, in marine mammals paly has an adptive function, preparing the individual for adult life.
\end{abstract}

Key words: Tucuxi, Play, Foraging abilities, Immature animals.

omportamentos de brincadeira são fáceis de identificar, mas difíceis de definir (Rasa, 1984; Spinka, Newberry \& Bekoff, 2001). Geralmente sua ocorrência é mais freqüente em animais jovens, apresentando algumas características comuns: falta de função aparente, utilização de comportamentos específicos da espécie de forma exagerada em intensidade ou número de repetições, podendo ser misturados a padrões apropriados de 
comportamento. Estudos sobre a brincadeira sugerem três possíveis funções: desenvolvimento da flexibilidade comportamental calibrada pelo meio ambiente; promoção de habilidades cognitivas e motoras; promoção do reconhecimento de parentes e da aprendizagem social (Fagen, 1981; Smith, 1982).

Este tipo de comportamento não ocorre em todas as espécies animais; sugere-se que ele aparece associado à vida social e ao alto grau de desenvolvimento encefálico. Todos as espécies estudadas de mamíferos, porém, o apresentam. Spinka et al. (2001) sugerem que existe uma unidade funcional e filogenética subjacente à brincadeira em todos os mamíferos, e que ela serviria, basicamente, como um treino para situações inesperadas, sejam motoras ou emocionais. Esta última proposta considera que a brincadeira, principalmente aquela que tem componentes motores, traz benefícios imediatos. Os benefícios funcionais da brincadeira, como de qualquer outro comportamento, são mais difíceis de avaliar. Spinka et al. (2001) sugerem que esses benefícios são pouco importantes, pois em condições adversas a brincadeira tende a diminuir ou até mesmo a desaparecer (Barret, Dunbar \& Dunbar, 1992), porém outros autores sugerem que benefícios de longo prazo advêm da brincadeira, especialmente aquelas que permitem treinar padrões comportamentais que devem ser aperfeiçoados para que filhotes possam se alimentar e/ ou se defender de forma independente (Mann \& Smuts, 1999).

Cetáceos possuem todas as características para a ocorrência de brincadeira: são mamíferos com alto grau de encefalização, tem vida social complexa e necessitam aprender um repertório comportamental complexo, incluindo interações com presas, que apresentam alto grau de imprevisibilidade (Connor, Mann, Tyack \& Whitehead, 1998). A brincadeira já foi descrita em algumas espécies de cetáceos: orcas (Baird, 2000; Guinet, 1991), golfinhos nariz-de-garrafa (Mann \& Smuts, 1999; McCowan, Marino, Vance, Walke \& Reiss, 2000) e belugas (Delfour \& Aulagnier, 1997). De uma maneira geral, no entanto, esta é uma ordem pouco estudada do ponto de vista comportamental, e o número de espécies está, certamente, subestimado. Nós estudamos a brincadeira em uma espécie pouco conhecida, o boto cinza (Sotalia fluviatilis), que habita a costa das Américas do Sul e Central (Carr, 2000; da Silva \& Best, 1996).

\section{Método}

Acompanhamos grupos naturais de boto cinza, que ocorriam na Praia de Pipa, RN. As observações foram feitas de um ponto fixo localizado no Hotel Village da Natureza, tendo à frente a enseada dos golfinhos da
Praia do Madeiro, em Pipa (cerca de $80 \mathrm{~km}$ de Natal, pertencente ao município de Tibau do Sul). As observações foram realizadas por dois pesquisadores treinados, com a ajuda de binóculos, com registros em um gravador. Os pesquisadores passaram por um treinamento até que o índice de concordância na identificação das categorias comportamentais fosse de pelo menos $85 \%$.

Os animais foram observados por 41 dias, entre os meses de janeiro de 2000 e setembro de 2001 (18 meses), por seis horas diárias, totalizando 246 horas de observação. Comportamentos de brincadeira foram observados em 19 dias (46,34\% dos dias).

Quando um grupo era avistado na enseada, contávamos o número de animais presentes, identificávamos a faixa etária a que pertenciam (ver definição abaixo) e registrávamos, quando ocorriam, os diferentes tipos de comportamento e qual a faixa etária do animal que os realizava.

\section{Definições utilizadas}

Faixa etária: os animais foram classificados como adultos e imaturos. Foram considerados como imaturos aqueles animais que apresentavam cerca de $1 / 3$ a $3 / 4$ do tamanho adulto. Para a classificação dos indivíduos nessas faixas etárias era necessária a concordância entre os observadores quanto ao tamanho aproximado do animal.

Grupo de animais: o critério utilizado neste trabalho foi espacial, sendo considerado como grupo todos os animais que estivessem ao mesmo tempo utilizando a área interna da enseada, mesmo quando afastados uns dos outros ou realizando atividades diferentes. Este critério foi o preferido porque a observação continuada permitiu verificar que esses animais tendiam a se aproximar e executar atividades conjuntas em diferentes momentos, durante a permanência na enseada, e também chegavam ou deixavam juntos a enseada.

Grupo de brincadeira: foram considerados como fazendo parte de um mesmo grupo de brincadeira, os animais que estivessem bastante próximos uns dos outros, com aproximadamente um a dois corpos de distância e engajados em algum dos diferentes tipos de brincadeiras que caracterizamos para esta espécie. Um dos critérios para considerar o grupo como de brincadeira era a presença de pelo menos um animal imaturo.

Comportamento de brincadeira: consideramos como brincadeira todos os comportamentos, dentre os observados, que atendiam os seguintes critérios: desempenhado por ou em grupo que contivesse animais imaturos; ausência de função aparente; repetição exagerada, em intensidade ou freqüência, de comportamentos exibidos por animais adultos. O término de um episódio de brincadeira foi definido como a não apresentação deste comportamento após cinco minutos do último registro. 
Freqüência de ocorrência dos tipos de brincadeira

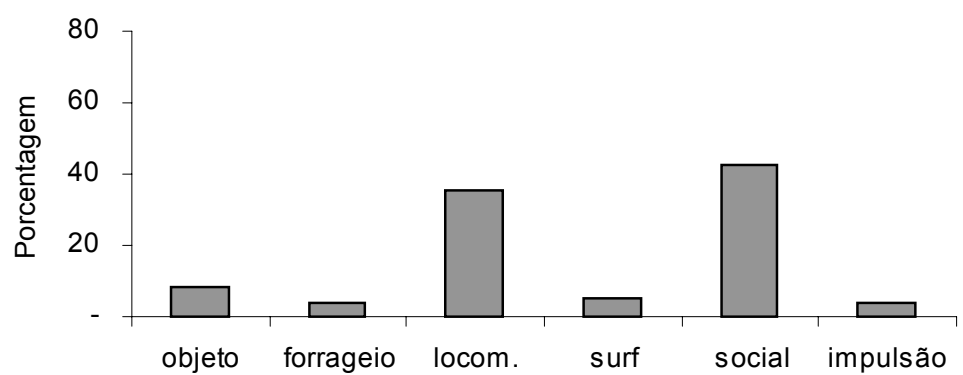

Figura 1. Porcentagem de freqüência de ocorrência dos seis tipos de brincadeira durante todo o período de observação.

\section{Resultados}

A análise dos comportamentos registrados durante as observações resultou na proposição de seis categorias comportamentais de brincadeira: brincadeira com objeto, brincadeira de forrageio, brincadeira locomotora, surf, brincadeira social e brincadeira de impulsão, com freqüências diferenciadas, tal como descrito na Figura 1.

$\mathrm{O}$ número médio de animais na enseada, aquilo que caracterizamos como grupo, foi de 4,2. Porém o número médio do grupo de brincadeira foi menor, 2,5, sugerindo que nem todos os animais participavam da brincadeira durante o período de observação. De fato, a presença dos adultos nos grupos foi freqüente, mas a participação na brincadeira foi mais discreta (Fig. 2). A composição dos grupos de brincadeira variou em função do tipo de brincadeira observada (Fig. 3), sendo predominante o animal só na brincadeira com objeto e de forrageio, e a presença de adultos no surf e nas brincadeiras social e de impulsão.

Os tipos de brincadeira identificados são descritos abaixo e as descrições gráficas foram incluídas na Figura 4:

1. Brincadeira com objetos: neste tipo de brincadeira, os animais utilizam objetos que estejam boiando na água (algas, gravetos, sacos plásticos, etc.), jogando-os para o alto com a boca ou com a nadadeira caudal, carregando-os com o dorso, ou nadando e segurando-os com a boca (Fig. 4a, b, c e d);

2. Brincadeira de forrageio: caracterizada quando animais imaturos, sozinhos ou acompanhados, perseguem pequenos peixes, próximo à zona de arrebentação, local de alimentação dos animais. Este comportamento foi considerado como brincadeira porque animais imaturos nunca foram vistos capturando com sucesso a presa (Fig. 4f);

\section{Composição do grupo $\quad$ GGT}

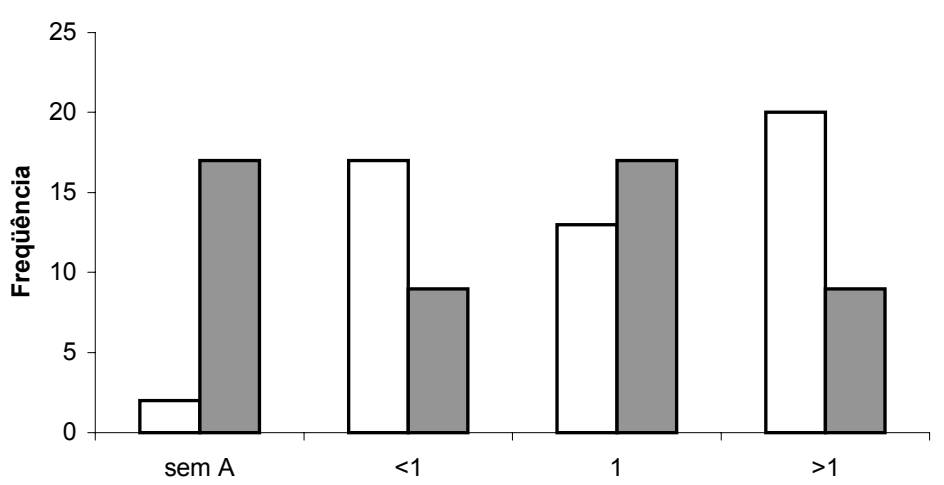

Figura 2. Freqüência de ocorrência de grupos na enseada (GT - grupo total) nas colunas brancas e de grupos de brincadeira (GB) nas colunas cinzas, sem a presença de adultos, com a proporção adultos/imaturos menor que um $(<1)$, igual a um (1) e maior que um $(<1)$. 


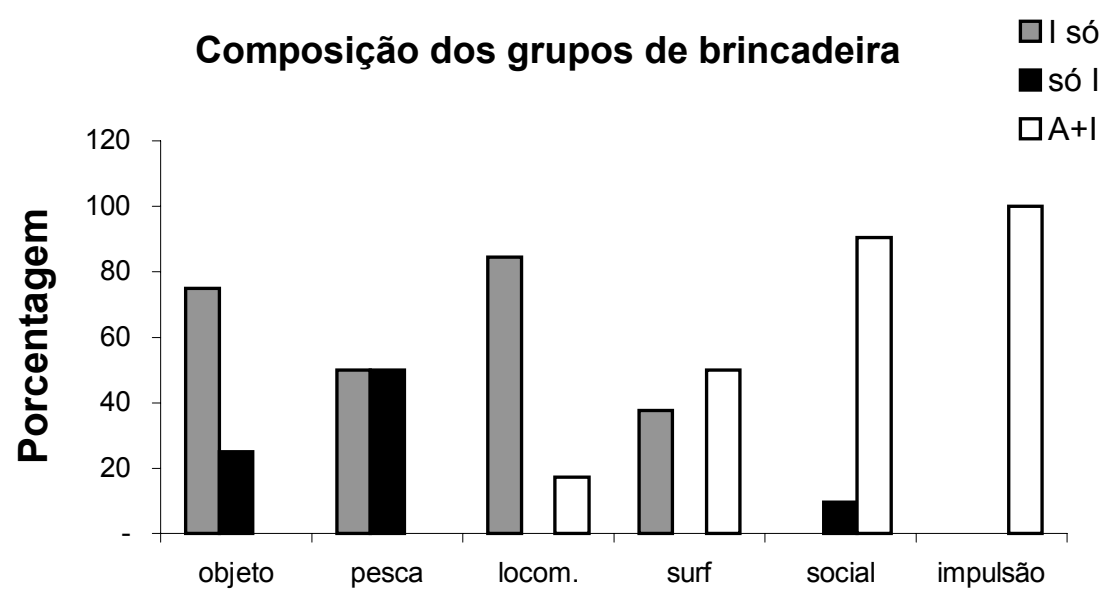

Figura 3. Distribuição da composição dos grupos de brincadeira em grupos com apenas um animal imaturo (I) nas barras cinzas, grupos só de imaturos (só I) nas barras pretas, e grupos com adultos e imaturos $(\mathrm{A}+\mathrm{I})$ nas barras brancas.

3. Brincadeira locomotora: foi considerado como brincadeira locomotora o conjunto de atividades aéreas realizadas pelos animais imaturos, tais como saltos, periscópios, batidas de cauda e cabeça e caídas. Essas atividades aéreas podiam ocorrer solitariamente ou, como visto mais freqüentemente, em grupos, onde saltos podiam ser vistos sendo realizados por pares de adultos e imaturos em sincronia. Estes saltos eram em geral apresentados durante os períodos que caracterizamos como de brincadeira social, mas suas freqüências foram registradas independentemente;

4. Surf: caracterizado como o deslocamento em que o animal, sozinho ou acompanhado, aproveita o movimento das ondas, podendo realizar saltos sincronizados. Este comportamento nunca foi visto associado com perseguições a peixes, o que o caracterizaria como atividade de forrageio, e por este motivo foi considerado como mais um tipo de brincadeira (Fig. 4e);

5. Brincadeira social: Neste tipo de brincadeira, sempre observada com os animais localizados muito próximos à superfície da água, os golfinhos são vistos numa intensa agitação locomotora, provocando turbulências na água e com bastante contato físico. É freqüente a observação de animais imaturos passando com seus corpos por cima de outros animais (adultos ou outros imaturos), algumas vezes batendo fortemente seu corpo contra o do outro animal, ou caindo por cima do outro depois de um salto. Os animais podem ser vistos ainda em perseguições mútuas, nadando em sincronia e dando leves mordiscadas uns nos outros;
6. Brincadeira de impulsão (calf tossing): caracterizado como o comportamento em que animais adultos jogam imaturos para o alto, ficando estes com o corpo todo fora da água (Fig. 4g).

\section{Discussão}

Não há registros detalhados sobre a brincadeira em estudos anteriores com o boto cinza. A maioria dos estudos refere-se a este comportamento de uma maneira geral, inespecífica. Geise (1991), por exemplo, define a brincadeira entre os golfinhos de $S$. fluviatilis como "um misto de vários comportamentos, como saltos, deslocamentos rápidos, toques e mordiscadas mútuas" (p. 33). Nossos resultados sugerem que a brincadeira ocorre de forma diversificada em botos cinza, sendo mais freqüente em animais imaturos. Este trabalho permitiu detalhar melhor a caracterização do comportamento de brincadeira nesta espécie, classificando-os em categorias diferenciadas quanto à execução e possíveis funções. Nossos dados estão em acordo com os dados da literatura referentes a outras espécies de cetáceos, como o golfinho narizde-garrafa (Mann \& Smuts, 1999; McCowan et al., 2000), a orca (Guinet, 1991; Guinet \& Bouvier, 1995) e o golfinho rotador (Johnson \& Norris, 1994).

As formas mais freqüentes de brincadeira foram a locomotora e a brincadeira social. As outras foram observadas em freqüência muito menor. Os dois primeiros tipos de brincadeira, no entanto, são mais aparentes do que os outros, e talvez tenham sido mais facilmente observados e registrados. É importante lembrar que esses 

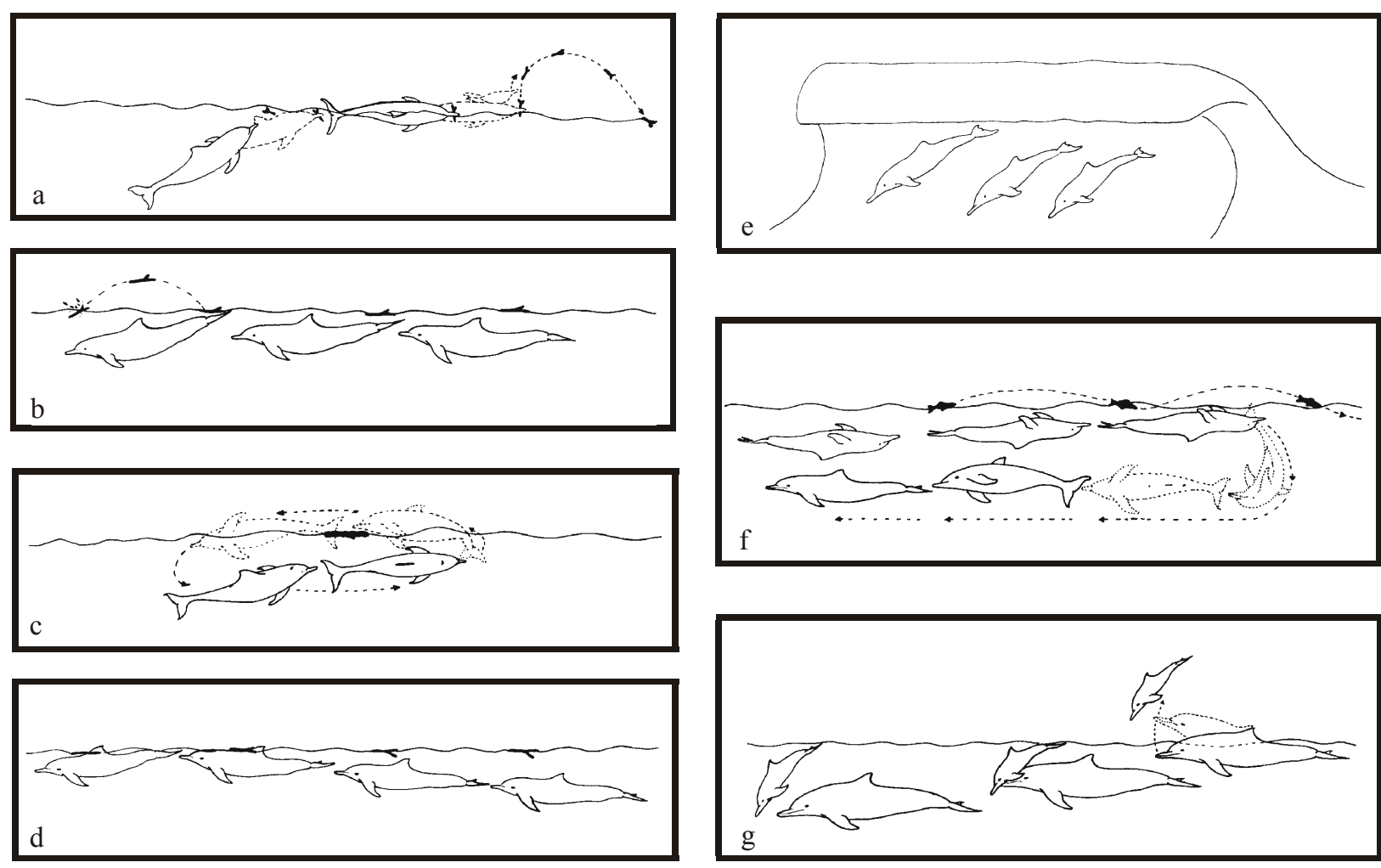

Figura 4. Representação gráfica dos movimentos dos animais durante a brincadeira com objeto (a, b, c e d), surf (e), brincadeira de forrageio (f) e impulsão do filhote (g).

comportamentos ocorrem em um espaço de tempo muito curto e em ambiente nem sempre favorável à sua observação, podendo levar a tais desvios.

Diferentes funções têm sido atribuídas aos diferentes tipos de brincadeira e há consenso de que este tipo de comportamento é benéfico aos animais envolvidos (Fagen, 1981; Smith, 1982; Spinka et al, 2001), apesar dos custos envolvidos (Caro, 1987). No entanto, há discordância quanto ao atendimento a uma ou várias funções adaptativas e aos benefícios, se imediatos ou de longo prazo. Recentemente, Spinka e colaboradores (2001) sugeriram uma função única para a brincadeira em mamíferos, que seria o treinamento para o inesperado, tanto do ponto de vista motor quanto emocional. De uma certa forma esses autores reúnem os pontos de vista anteriores, na medida em que: a. superam a questão do benefício retardado, pois acreditam que em espécies nas quais os adultos têm que lidar com situações inesperadas, como espécies presa ou predadora, a brincadeira, principalmente a social, não desaparece ao final da imaturidade mas permanece durante a idade adulta; b. propõem benefícios de curto prazo para a brincadeira motora, que proporcionaria aumento da versatilidade de movimentos permitindo a recuperação de choques "gravitacionais", "cinemáticos" ou "posturais" e de longo prazo para a brincadeira social, que permitiria ao indivíduos lidar com choques "psicológicos" ou cognitivos imediatos e que ocorrem mais tarde na vida.

A divisão dos tipos de brincadeira que descrevemos para o boto cinza entre locomotora e social resulta em três tipos claramente locomotores (brincadeira com objeto, de forrageio e locomotora) dois tipos claramente sociais (brincadeira social e de impulsão) e um tipo intermediário (surf). Tal como previsto por Spinka et al. (2001) para espécies com comportamento complexo e alto grau de encefalização, como é o caso dos cetáceos (Connor et al., 1998), os adultos se envolvem mais nas brincadeiras sociais e praticamente não mostram envolvimento com as locomotoras. Porém, adultos estão sempre presentes durante a ocorrência da brincadeira, mesmo quando não participam, o que sugere que, para que ocorra, a brincadeira necessita de ambientes seguros, neste caso garantidos pela proteção natural das enseadas e pela presença de adultos que podem prover vigilância e proteção.

Entre as brincadeiras motoras, aquela com objetos é a mais extensamente descrita em cetáceos (Delfour \& Alagnier, 1997; Johnson \& Norris, 1994; Mann \& Smuts, 1999; McCowan et al., 2000). Os relatos sugerem que ela 
ocorre com animais muito jovens que, aparentemente, testam seus limites motores e aperfeiçoam habilidades posturais (Chalmers \& Locke-Haydon, 1984). Nas espécies que apresentam brincadeira solitária com objetos, tais comportamentos são muitos semelhantes aos comportamentos predatórios de animais adultos, o que sugere uma função de aprendizado (Smith, 1982).

Isto também parece ser verdadeiro para a brincadeira locomotora. No entanto, enquanto a primeira é basicamente solitária, a brincadeira locomotora pode envolver saltos sincronizados de mais de um animal, o que promove o desenvolvimento de ações coordenadas que serão necessárias na atividade de forrageio (Spinelli, Nascimento, Medeiros \& Yamamoto, 2001). Finalmente, a brincadeira de forrageio caracteriza de forma exemplar um dos critérios de definição da brincadeira, a imitação do comportamento adulto, porém sem completar sua função, em nosso caso, a pesca. A brincadeira como forma de treinamento para o comportamento predatório já foi descrito em carnívoros (Pellis \& Dewsbury, 2000). Em cetáceos, o forrageio tem caráter especializado, e a descrição de imaturos envolvendo-se em atividades que lembram o forrageio de adultos ou técnicas específicas de forrageio já foi descrito em golfinhos nariz-de-garrafa (Mann \& Smuts, 1999) e em orcas (Guinet, 1991; Guinet \& Bouvier, 1995), sem porém resultar em apreensão de presas. Nesta última, filhotes, sempre acompanhados de adultos, foram vistos encalhando o corpo na praia, de forma semelhante a adultos quando caçam focas. Neste caso, porém, o comportamento era feito na ausência de presas, portanto sem que a função esperada do comportamento, a captura da presa, se completasse (Guinet, 1991).

Os grupos de brincadeira social no boto cinza tiveram a participação de adultos em cerca de $90 \%$ das ocorrências. Esta é uma proporção muito maior do que o observado em outras espécies, nas quais a participação dos adultos é ocasional (Gomendio, 1988; Smith, 1982), embora tenha sido observada em cetáceos (Mann \& Smuts, 1999). A razão para a alta taxa de participação de adultos pode se dever à composição dos grupos, com pequeno número de filhotes, ou à participação passiva dos adultos, como observado em Callithrix jacchus (observ. pes.). A brincadeira de impulsão, um tipo especial de brincadeira social, só foi observada entre adultos e filhotes. Aparentemente, a força física necessária para a impulsão de um filhote requer a participação do animal de maior porte.

Em conclusão, a brincadeira em S. fluviatilis, é similar à de mamíferos terrestres, apresentando as mesmas características de participação preferencial de imaturos, repetição e ausência aparente de função. Além disso, estes dados sugerem que, à semelhança dos mamíferos terrestres, também em mamíferos aquáticos a brincadeira tem função adaptativa, preparando o indivíduo para a vida adulta e para desafios presentes no momento de seu desenvolvimento.

\section{Agradecimentos}

Durante a duração do estudo L.H.P.S. e M.E.Y. receberam bolsas do CNPq (PIBIC-UFRN e \#524409/96, respectivamente) apoio financeiro do CNPq e da CAPES. Os autores desejam agradecer também P.I.A.P. Medeiros pelo auxílio com a parte gráfica, a David Hasset pela cessão de sua casa durante o trabalho de campo, e a população de Pipa, em especial V. M. Rebello, pelo suporte durante a coleta de dados.

\section{Referências}

Baird, R. W. (2000). The killer whale: Foraging specializations and group hunting. In J. Mann, R. C. Connor, P. L. Tyack \& H. Whitehead (Orgs.), Cetacean Societies (pp. 127-153). Chicago: University of Chicago Press.

Barret, L., Dunbar, R. I. M., \& Dunbar, P. (1992). Environmental influences on play behaviour in immature gelada baboons. Animal Behavior, 44, 111-115.

Caro, T. M. (1987). Indirect costs of play: Cheetah cubs reduce maternal hunting success. Animal Behavior, 35, 295-297.

Carr, T. (2000). Tucuxi (Sotalia fluviatilis) occurs in Nicaragua, $800 \mathrm{~km}$ north of previously known range. Marine Mammal Science, 16, 447452.

Chalmers, N. R., \& Locke-Haydon, J. (1984). Correlations among measures of playfulness and skilfulness in captive common marmosets (Callithrix jacchus jacchus). Developmental Psychobiology, 17, 191-208.

Connor, R. C., Mann, J., Tyack, P. L., \& Whitehead, H. (1998). Social evolution in toothed whales. Trends in Ecology and Evolution (TREE), $13,228-232$

da Silva, V. M. F., \& Best, R. C. (1996). Mammalian species: Sotalia fluviatilis. Mammalian Species, 527, 1-7.

Delfour, F., \& Aulagnier, S. (1997). Bubbleblow in beluga whales (Delphinapterus leucas): a play activity? Behavioral Processes, 40, 183-186.

Fagen, R. (1981). Animal Play Behavior. Oxford: Oxford University Press. Geise, L. (1991). O comportamento dos golfinhos. Ciência Hoje, 77, 2733.

Gomendio, M. (1988). The development of different types of play in gazelles: implications for the nature and function of play. Animal Behavior, 36, 825-836.

Guinet, C. (1991). Intentional stranding apprenticeship and social play in killer whales (Orcinus orca). Canadian Journal of Zoology, 69, 27122716.

Guinet, C., \& Bouvier, J. (1995). Development of intentional stranding hunting techniques in killer whale (Orcinus orca) calves at Crozet Archipelago. Canadian Journal of Zoology, 73, 27-33.

Johnson, C. M., \& Norris, K. S. (1994). Social behavior. In K. S. Norris, B. Würsig, R. S. Wells, \& M. Würsig (Orgs.), The Hawaiian Spinner Dolphin (pp. 243-286). Berkeley: University of California Press.

Mann, J., \& Smuts, B. B. (1999). Behavioral development in wild bottlenose dolphin newborns (Tursiops sp.). Behaviour, 136, 529-566.

McCowan, B., Marino, L., Vance, E., Walke, L., \& Reiss, D. (2000). Bubble ring play of bottlenose dolphins (Tursiops truncatus): 
Implications for cognition. Journal of Comparative Psychology, 114, 98-106.

Pellis, V. C., \& Dewsbury, D. A. (2000). The organization of play fighting in the grasshopper mouse (Onychomys leucogaster): Mixing predatory and sociosexual targets and tactics. Agressive Behavior, 26, 319-334.

Rasa, O. A. E. (1984). A motivational analysis of object play in juvenile dwarf mongooses (Helogale undulata rufula). Animal Behavior, 32, 579-589.

Smith, P. K. (1982). Does play matter? Functional and evolutionary aspects of animal and human play. Behavioral and Brain Sciences, 5, 139-184.
Spinka, M., Newberry, R. C., \& Bekoff, M. (2001). Mammalian play: Training for the unexpected. Quarterly Review of Biology, 76, 141168.

Spinelli, L. H. P., Nascimento, L. F., Medeiros, P. I. A. P., \& Yamamoto, M. E. (2001). Interações entre adultos e imaturos em Sotalia fluviatilis: Evidência de cuidado alomaterno? Anais de Etologia, 19, 290.

Luísa Helena Pinheiro Spinelli é aluna de mestrado do Programa de Pós-Graduação em Psicobiologia da Universidade Federal do Rio Grande do Norte.

Lídio França do Nascimento é mestre em Psicobiologia pela Universidade Federal do Rio Grande do Norte.

Maria Emília Yamamoto, doutora em Psicofarmacologia pela Universidade Federal de São Paulo, SP e pós-doutorado pela University of Reading (Reino Unido), é professora titular do Departamento de Fisiologia da Universidade Federal do Rio Grande do Norte.

Endereço para correspondência: Maria Emília Yamamoto, Caixa Postal 1511, 59078-970, Natal, RN. Telefone (084)215.3409. Fax (084)211.9206. E-mail: emilia.yamamoto@uol.com.br. 